Murray, R.W., Miller, D.J., and Kryc, K.A.

ODP Technical Note 29

\section{ANALysis Of MAJOR AND TRACE Elements IN ROCKS, SEDIMENTS, AND INTERSTITIAL WATERS BY INDUCTIVELY COUPLED Plasma-ATOMIC EMisSion SPECTROMETRY (ICP-AES) ${ }^{1,2}$}

\author{
R.W. Murray, ${ }^{3}$ D. Jay Miller, ${ }^{4}$ and K.A. Kryc $^{3}$
}

\section{INTRODUCTION}

This Technical Note is designed to offer principles and guidelines for geochemical analysis by inductively coupled plasma-atomic emission spectrometry (ICP-AES) on board the Ocean Drilling Program (ODP) drillship JOIDES Resolution. The goal is to provide background instructions so that various types of samples (including igneous rocks, sedimentary rocks, sediments, and interstitial waters) may be analyzed for a comprehensive suite of major and trace elements. This Technical Note is not intended to be a stand-alone document explaining all the parameters of ICP-AES (appropriate references to that end are supplied below), but instead we hope to provide the fundamentals of sample preparation, analysis, and data reduction, resulting in high-quality shipboard analytical data. The critical advantage of ICP-AES is that rapid and quantitative analysis of a variety of sample types can be conducted relatively easily with a single instrument.

Care has been taken to ensure that these instructions are not overly instrument or software specific. The sections on basic ICP principles, sample preparation, recommended wavelengths, and the like are not unique to the Jobin-Yvon JY2000 instrument currently on board the Resolution. Where necessary, however, instrument-specific guidelines are provided. Accordingly, several passages in this technical note have been based on the owner's manual provided by Jobin-Yvon (JY), manu-
${ }^{1}$ Murray, R.W., Miller, D.J., Kryc, K.A., 2000. Analysis of major and trace elements in rocks, sediments, and interstitial waters by inductively coupled plasma-atomic emission spectrometry (ICP-AES). ODP Tech. Note, 29 [Online]. Available from World Wide Web: $<$ http://www-odp.tamu.edu/ publications/tnotes/tn29/INDEX.HTM>. [Cited YYYY-MM-DD]

${ }^{2}$ See Disclaimer, p. 23.

${ }^{3}$ Department of Earth Sciences, Boston University, Boston MA 02215, USA. rickm@bu.edu

${ }^{4}$ Ocean Drilling Program, Texas A\&M University, 1000 Discovery Drive, College Station TX 77845-9547, USA. 
R.W. MURRAY ET AL.

facturer of the JY2000, as well as on notes from the Boston University Analytical Geochemistry Laboratory. A companion booklet of detailed software notes from Boston University has also been provided to the ODP Chemistry Laboratory. Additional information may be found in the "Explanatory Notes" of the Legs 187-189 Initial Reports volumes of the Proceedings of the Ocean Drilling Program (Shipboard Scientific Party, in press $a$, in press $b$, in press $c$ ) because these legs were the first to use the shipboard ICP-AES for igneous rock (Leg 187) and interstitial water (Legs 188 and 189) analyses.

A list of ICP-AES references is included in "Useful References Discussing ICP-AES Techniques," p. 19, copies of which are available on board the Resolution. Although in some cases there is more information in these publications than is necessary to operate the JY2000 instrument, readers are encouraged to become familiar with the content to enable them to customize key aspects of the sample preparation and analytical procedures to best meet project-specific needs. Furthermore, the methodologies and procedures described here do not maximize the capabilities of the instrument, and individual shipboard scientists may choose to target particular chemical elements not discussed below. These publications will assist them in their endeavors.

\section{BASIC OVERVIEW OF ICP-AES}

ICP-AES is an emission spectrophotometric technique, exploiting the fact that excited electrons emit energy at a given wavelength as they return to ground state. The fundamental characteristic of this process is that each element emits energy at specific wavelengths peculiar to its chemical character. Although each element emits energy at multiple wavelengths, in the ICP-AES technique it is most common to select a single wavelength (or a very few) for a given element. The intensity of the energy emitted at the chosen wavelength is proportional to the amount (concentration) of that element in the analyzed sample. Thus, by determining which wavelengths are emitted by a sample and by determining their intensities, the analyst can quantify the elemental composition of the given sample relative to a reference standard.

ICP-AES analysis requires a sample to be in solution. Thus, interstitial waters can be analyzed simply, requiring only dilution in most cases. Igneous rocks, sedimentary rocks, and sediments, however, must be dissolved. This can be achieved either by a combined acid attack employing $\mathrm{HF}, \mathrm{HNO}_{3}$, and $\mathrm{HCl}$ acids, or by a $\mathrm{LiBO}_{2}$ flux-fusion technique similar to that used for XRF preparation. In addition to being somewhat dangerous for routine shipboard work (because of the highly reactive nature of $\mathrm{HF}$ ), the acid attack is not able to generate consistent and reliable data for Si because it volatilizes in the presence of HF. The acid digestion procedure also often results in incomplete analysis of refractory elements such as $\mathrm{Ti}, \mathrm{Cr}$, and $\mathrm{Zr}$ because their host minerals are often difficult to dissolve. The flux-fusion approach is employed on board the Resolution for several reasons: (1) it is safer because HF is not involved; (2) it is a complete dissolution technique, allowing determination of all elements, including Si and the refractory elements; (3) the resultant solutions are similar in composition (or matrix) because they are dominated by the presence of the $\mathrm{LiBO}_{2}$ flux; and (4) the solutions are stable in dilute $\mathrm{HNO}_{3}$ acid and can be transported safely back to the scientist's laboratory for further shore-based study. 
R.W. MURRAY ET AL.

All ICP-AES systems consist of several components. We shall focus this discussion on three main aspects: the sample introduction system, the torch assembly, and the spectrometer. The sample introduction system on the ICP-AES consists of a peristaltic pump, Teflon tubing, a nebulizer, and a spray chamber. The fluid sample is pumped into the nebulizer via the peristaltic pump. The nebulizer generates an aerosol mist and injects humidified Ar gas into the chamber along with the sample. See "Nebulizers," p. 7, for a more detailed discussion of nebulizers. This mist accumulates in the spray chamber, where the largest mist particles settle out as waste and the finest particles are subsequently swept into the torch assembly. Approximately $1 \%$ of the total solution eventually enters the torch as a mist, whereas the remainder is pumped away as waste.

Humidification of the Ar gas injected into the nebulizer is important when analyzing samples with high dissolved solids, as is often the case with analysis of ODP rocks, sediments, and interstitial waters. Humidification takes place in the Ar humidifier, where Ar is bubbled through deionized water prior to its expulsion in the nebulizer.

The fine aerosol mist containing Ar gas and sample is injected vertically up the length of the torch assembly into the plasma. There are several recommended Ar flow rates used in the torch, as described in detail in the owner's manual and in the various publications provided. The radio frequency-generated and maintained Ar plasma, portions of which are as hot as $10,000 \mathrm{~K}$, excites the electrons. When the electrons return to ground state at a certain spatial position in the plasma, they emit energy at the specific wavelengths peculiar to the sample's elemental composition.

The plasma is viewed horizontally by an optical channel. Light emitted from the plasma is focused through a lens and passed through an entrance slit into the spectrometer. There are two types of spectrometers used in ICP-AES analysis: sequential (monochromator) and simultaneous (polychromator). The JY2000 has a sequential spectrometer. This means that the diffraction grating in the spectrometer is analogous to a prism that refracts visible light into its component colors. The detector (photomultiplier tube) is fixed in space at the far end of the spectrometer. Rotation of the diffraction grating sequentially moves each wavelength into the detector. The computer control ensures that the detector is synchronized with the grating so that the intensity at the detector at any given time is correlated with the wavelength being diffracted by the grating. The operator enters the wavelengths that he or she wishes to detect into the computer, the grating sequentially moves to the specified wavelengths, and the energy intensity at each wavelength is measured to provide a quantitative result that can be compared to a reference standard. Using standard spectroscopic techniques (e.g., background corrections), sequential ICP-AES can provide extremely flexible and rapid analysis of a number of chemical elements. The spectrometer is flushed with $\mathrm{N}_{2}$ gas to improve the detection limits of elements with emission wavelengths that are severely compromised by interference with air (e.g., P). This $\mathrm{N}_{2}$ flush, which is constantly maintained in the instrument regardless of whether such elements are being analyzed, also protects the optics from the corrosive aspects of the atmosphere, which are particularly acute at sea. 


\section{MASS, VOLUME, AND UNITS}

Throughout the following sections, all units and dilution factors of rocks and sediments (i.e., materials that are initially solid) are given on a per mass basis, and all units and dilution factors for interstitial waters are given on a per volume basis. This allows the ease of working with these different matrices on a weight vs. volume basis, and also follows standard conventions (e.g., $\mu \mathrm{M}$ in interstitial water data is given in micromoles per liter, that is, per volume).

Acid concentrations in analyte solutions are given in percentage (\%) units, as opposed to molarity (M). Although either unit system is appropriate and equally accurate, much of the ICP-AES literature refers to acid concentrations in percentage units, and so that convention is followed here. Concentrated $\mathrm{HNO}_{3}$ acid $(16 \mathrm{M})$ is $70 \% \mathrm{HNO}_{3}$. Therefore, preparation of $10 \% \mathrm{HNO}_{3}$ (by volume) involves a $7 \times$ dilution of concentrated acid (e.g., $1 \mathrm{~mL}$ of concentrated acid added to $6 \mathrm{~mL}$ of deionized water $[\mathrm{DI}])$.

Concentrations of the 10 major elements in rocks and sediments are commonly given in weight percent units of the oxide (e.g., $\mathrm{SiO}_{2}$ ), whereas in calibration standards and prime standards purchased from a vendor such as Fisher or VWR the concentrations are sometimes given in $\mu \mathrm{g} / \mathrm{mL}$ (ppm) for an element (e.g., Si). Table $\mathbf{T} 1$ provides conversion factors between oxide and elemental concentrations. (These are multiplicative factors; to convert $\mathrm{SiO}_{2}$ to $\mathrm{Si}$, multiply by 0.4675 .) The conversion from ppm to weight percent (part per hundred) involves division by $10^{4}$.

\section{SAMPLE PREPARATION}

\section{Overview}

There are three fundamental guiding principles of ICP-AES sample preparation that must be followed for every calibration and analysis, regardless of what is being analyzed (rocks, sediments, or interstitial waters).

1. Filtration: All solutions introduced to the instrument must be filtered. This is most efficiently performed through Acrodisc filters that fit over a syringe. Failure to filter every solution may lead to a partially or completely clogged nebulizer, resulting in poor analytical precision or analysis failure. Clogged nebulizers are also extremely difficult to clean (see "Basic Maintenance Suggestions," p. 18).

2. Matrix Matching: The term "matrix" refers to the sum of all compositional characteristics of a solution, including its acid composition. Calibration standards and samples must be matrix-matched in terms of composition, total dissolved solids (TDS), and acid concentration (in percent) of the solution. For analysis of solid materials that have been dissolved, the use of $\mathrm{LiBO}_{2}$ flux effectively means that the standards and samples have been compositionally matrix matched (as will be detailed in "Preparation of Rock/Sediment Samples," p. 6), but it is also important to have the standards and samples be of the same acid concentration. TDS refers to the total mass (in milligrams) of powder (sample plus flux) dissolved in a given mass of solution, and is commonly quantified as a function of dilution factor (see "Dilution Factors," p. 7). There is a wide range of acceptable acid concentrations, any one
T1. Conversion factors between oxide and elemental concentrations, p. 24. 
R.W. MURRAY ET AL.

of which is likely to suffice, but it is of critical importance that whatever acid concentration is used during a particular run, that it be the same for all standards and samples in that run. Typical concentrations for rock and sediment samples are $5 \%$ or $10 \% \mathrm{HNO}_{3}$. For interstitial water analysis, the calibration standards must be prepared with the same background salt concentration.

3. Cleaning: Throughout the laboratory, great care and diligence must be exercised to keep sample bottles and ICP glassware used for the analysis of difference matrices separate. Because Li and B are of particular interest to interstitial water chemists, all sample bottles, autosampler vials and tubes, Teflon tubing, peristaltic pump tubing, and torch assembly glassware that are used during the analysis of rocks and sediments (and thus have been exposed to tremendous amounts of Li and B in the $\mathrm{LiBO}_{2}$ flux) must be kept separate from those items used in the preparation and analysis of interstitial waters. As importantly, bottles and glassware that have been used in the preparation and analysis of the $\mathrm{LiBO}_{2}$ flux solutions must be cleaned separately from interstitial water apparatus. Duplicate acid baths and other cleaning routines need to be maintained with the greatest rigor.

\section{Flux-Fusion Preparation of Rocks and Sediments}

The procedure used here is similar to that used previously on board the Resolution for major element X-ray fluorescence (XRF) analysis. Whereas in major element XRF the fused glass disk serves as a solid target for the X-ray beam, in ICP-AES preparation the glass bead is dissolved in $10 \% \mathrm{HNO}_{3}$, the solution is filtered and diluted, and the resultant aqueous solution is subsequently introduced into the ICP-AES for analysis.

In the following presentation, the analyst must decide whether to use ignited samples, depending on the analytical needs. It is most common to use ignited samples for igneous rocks, with the final analysis including a determination of loss on ignition (LOI). For sediments and sedimentary rocks, particularly carbonate-rich sediments, determination of LOI is often not performed, partly because LOI comprises a significant proportion of the total weight percent. Regardless, the following guidelines apply to the use of ignited or nonignited samples equally well. The most important consideration is that the proper sample and acid concentrations be used during calibration and analysis.

$\mathrm{LiBO}_{2}$ flux without La absorber, and indeed only ultrapure grade $\mathrm{LiBO}_{2}$, should be used. The heavy La absorber also contains other rare earth elements (REEs), and thus the standard and sample solutions will be compromised for future analyses, and the ICP-AES will be contaminated with background concentrations of REEs.

The most critical aspect of the preparation is the need to maintain a constant sample to flux ratio. This can be adjusted as needed on a case by case basis, but a ratio of $1: 4$ should suffice in most situations. ICPAES analysis requires a far smaller sample mass than XRF. Typically, 0.1 $\mathrm{g}$ of sample powder mixed with $0.4 \mathrm{~g}$ of $\mathrm{LiBO}_{2}$ flux will result in adequate solution for a thorough major and trace elemental analysis. However, if only limited sample mass is available (e.g., for the analysis of volcanic glasses), a smaller sample mass may be used, but the same sample:flux ratio must be maintained for all samples and calibration standards (otherwise the matrix will not match). For example, $0.05 \mathrm{~g}$ of 
R.W. MURRAY ET AL.

sample would require $0.2 \mathrm{~g}$ flux to maintain the suggested $1: 4$ ratio. Use of such smaller masses results in a smaller mass (volume) of analyte solution, but if a complete geochemical analysis is not required, very small sample masses (e.g., basaltic glass rims) can be analyzed for only a few target elements of high interest.

\section{Preparation of Rock/Sediment Samples}

1. Weigh $0.1 \mathrm{~g}$ of powdered, dried sample. ODP standard practice is to weigh within a range of $0.0995-0.1005 \mathrm{~g}$, with a precision of $0.5 \%$ of the measured value. If the sample weight falls within this range, weighing differences need not be accounted for during data reduction.

2. Mix the sample powder with $0.4 \mathrm{~g}$ of $\mathrm{LiBO}_{2}$ flux. In practice, this mass can be weighed out between 0.395 and $0.405 \mathrm{~g}$. Transfer the powder mix into a Pt-Au crucible, add $10 \mu \mathrm{L}$ of $0.172 \mathrm{mM} \mathrm{LiBr}$ wetting agent, and fuse at $1050^{\circ} \mathrm{C}$ for $10-12 \mathrm{~min}$. The wetting agent is prepared by dissolving $0.15 \mathrm{mg}$ of ultrapure LiBr powder into $10 \mathrm{~g}$ of DI.

3 . Let the bead cool and solidify in the bottom of the crucible. After cooling, pop the bead off the crucible bottom. A sharp "whack" squarely on the hard desktop facilitates release.

4. Dissolve the bead in $50 \mathrm{~mL}$ of $10 \% \mathrm{HNO}_{3}$ acid solution and shake. Dissolution is effective in a sonicator bath or a wrist-action shaker, and should take $\sim 45 \mathrm{~min}$ to $1 \mathrm{hr}$ in most cases. This solution is now at what is broadly termed a $100 \times$ dilution for TDS because the $0.5 \mathrm{~g}$ of flux + sample has been dissolved in $\sim 50 \mathrm{~g}$ of $\mathrm{HNO}_{3}(50 \mathrm{~g} / 0.5 \mathrm{~g}=100)$. This is also referred to as a $1 \%$ solution because $1 \%$ of the total mass consists of TDS. It is important to note that the dilution of the sample is significantly greater $(500 \times)$, because $0.1 \mathrm{~g}$ of powdered sample has been dissolved in $50 \mathrm{~g}$ of $\mathrm{HNO}_{3}(50 \mathrm{~g} / 0.1 \mathrm{~g}=500)$. The very small addition of $\mathrm{LiBr}$ does not significantly increase the TDS. The exact mass of the acid solution is not critical (i.e., $50 \mathrm{~mL} \mathrm{HNO}_{3}$ is $\sim 50 \mathrm{~g}$ ), but it is extremely important that the unknown samples, blanks, replicates, etc., be prepared identically to the rock SRMs used for calibration (see "Dilution Factors," p. 7).

5. After complete dissolution of the bead, the solution should be filtered. This is best achieved by aspirating $\sim 20 \mathrm{~mL}$ of the solution into a syringe and filtering through an Acrodisc into a cleaned scintillation vial. The remaining unfiltered solution can be saved for additional analysis, replicate analysis, or transport to a shorebased laboratory.

6 . The final analyte solution is prepared by pipetting a $5-\mathrm{mL}$ aliquot of the filtered solution and diluting it with $35 \mathrm{~mL}$ of $10 \% \mathrm{HNO}_{3}$ (to a total of $40 \mathrm{~mL})$. This is an additional $8 \times$ dilution $(40 \mathrm{~mL} / 5 \mathrm{~mL}=8)$ of the original $500 \times$ diluted solution described in Step 4 above (to arrive at a total $4000 \times$ dilution of the sample [not of the sample + flux]). This solution is dilute enough to enable use of a type-C Meinhard concentric nebulizer (see "Nebulizers," p. 7).

7. An analytical procedural blank is prepared identically to the samples, with the exception that only $0.4 \mathrm{~g}$ of flux is fused and dissolved. An additional $0.1 \mathrm{~g}$ of flux is not added to mimic the TDS of the $0.5 \mathrm{~g}$ mix of sample + flux because this would provide an inaccurate quantitation of the impurities introduced by the amount of flux used in preparation of the unknowns. 
R.W. MURRAY ET AL.

Preparation of Interstitial Waters

1. After $\mathrm{HNO}_{3}$ acidification of an aliquot of interstitial water sample, the preparation of interstitial water for ICP-AES analysis primarily involves dilution. Because the pore-water solutions have already been filtered during squeezing, additional filtration is not required.

2. The most common dilution for interstitial water samples is $10 \times$. Depending on the analysis speed and the number of elements analyzed, one can usually generate enough analyte with $0.5 \mathrm{~mL}$ of interstitial water diluted with $4.5 \mathrm{~mL}$ of DI (for a total analyte of $5.0 \mathrm{~mL}$ ). A $1-\mathrm{mL}$ sample $+9 \mathrm{~mL}$ DI solution allows for replicate analysis. In some cases, undiluted interstitial water may be analyzed, but care must be taken to not clog the nebulizer, as the likelihood of salt buildup at the nebulizer orifice is greatly increased.

3. An analytical blank is prepared identically by analyzing pure DI acidified to matrix match the samples. Be sure to use DI from the same bottle as used during dilution of the samples.

\section{GUIDELINES TO ANALYTICAL OPERATING CONDITIONS}

This section describes some basic considerations and guidelines for analytical conditions to be used during ICP-AES analysis.

\section{Dilution Factors}

\section{Rocks and Sediments}

The dissolution procedure described in "Flux-Fusion Preparation of Rocks and Sediments," p. 5, results in an aqueous solution that can be analyzed in a single analytical session for major and trace elements. It represents a $4000 \times$ (nominal) dilution of the sample. This dilution factor is appropriate for igneous rocks, sedimentary rocks, and sediments. We suggest working from this $4000 \times$ dilution as a baseline. Should there be leg-specific analytical requests, different dilutions can be prepared, as long as the overall goal of generating enough analyte at an appropriate concentration is fulfilled. See additional discussion in "Nebulizers," p. 7. As always, prepare the standard reference materials used to calibrate the ICP-AES at the same dilution factor and acid concentration as the unknown samples.

\section{Interstitial Waters}

As mentioned previously, the most common dilution factor for interstitial waters is a simple $10 \times$ dilution in DI. Undiluted seawater may be analyzed, particularly when low concentration elements are to of concern, but salting of the nebulizer must be monitored carefully. Use of the Ar humidifier will improve results for undiluted seawater analysis.

\section{Nebulizers}

In all circumstances, great care must be taken to avoid nebulizer clogging. Thus, all samples and standards should be filtered prior to analysis. Clogging can result from either a physical blockage of the small capillary inside the nebulizer or from the formation of a precipitate 
R.W. MURRAY ET AL.

around the ejection orifice. Nebulizer clogging can be easily diagnosed when the nebulizer Ar flow rate decreases simultaneously with an increase in Ar backpressure, which signals inhibition of $\mathrm{Ar}$ transport through the nebulizer. When the nebulizer begins to clog, the stability of the analysis greatly decreases and drift becomes insurmountable. The analytical run must be discontinued, the instrument shut down, and the nebulizer cleaned before the run can be resumed. It is very difficult to clean a nebulizer, and it can become tremendously time consuming to have to repeatedly deal with such clogging (see "Basic Maintenance Suggestions," p. 18).

A great deal of effort goes into selecting the proper nebulizer for a particular application. The most commonly used nebulizer is a concentric nebulizer (commonly referred to as a Meinhard nebulizer although other companies also manufacture them). See the literature on board the ship for a description of how this nebulizer operates. Meinhard and other concentric nebulizers provide excellent stability and signal to noise ratios, although they are more prone to clogging than other nebulizer types. Wide orifice type-C concentric nebulizers are appropriate for use with rocks and sediments as well as with interstitial water samples. For the dilutions described above, these nebulizers work very well, with minimal clogging, as long as proper cleaning procedures are followed.

ODP has also acquired several V-groove nebulizers, which allow for the analysis of samples with very high TDS. This type of nebulizer requires a slightly higher sample aspiration speed (controlled through the peristaltic pump) than a concentric nebulizer and thus consumes more analyte solution; however, the flexibility to easily analyze variable TDS solutions makes this nebulizer particularly well suited for very high TDS operations. It is important to note, however, that signal stability is significantly poorer for V-groove nebulizers, and they should only be used in exceptional circumstances. Testing of type-C concentric and V-groove nebulizers during Leg 187 and at Boston University documents that the concentric nebulization scheme provides a greater signal, better stability, and less noise for the analysis of rocks, sediments, and interstitial waters, at the dilution factors suggested here for routine operation.

\section{Stability of Solutions}

\section{Rocks and Sediments}

After a period of time, flux-fusion solutions may become unstable resulting in the precipitation of major and trace elements or the formation of a gel. These are not always immediately visible (the gel is clear), so solutions must be visually inspected carefully prior to analysis. An unstable solution must be discarded because the gel will strip dissolved trace metals from the analyte.

The stability of a solution is directly proportional to the dilution factor and acid content, and inversely proportional to the $\mathrm{SiO}_{2}$ content. A dilute solution is more stable than a concentrated one, and there is likely to be a noticeable difference in the shelf life of a flux-fusion solution prepared at $100 \times$ TDS vs. $1000 \times$ TDS. Likewise, a solution prepared in a $10 \% \mathrm{HNO}_{3}$ matrix is more stable than one prepared in $1 \% \mathrm{HNO}_{3}$. Conversely, a flux-fusion solution resulting from the dissolution of diatomaceous ooze or rhyolite (either of which are enriched in $\mathrm{SiO}_{2}$ ), is likely to be less stable than a solution prepared from a low-Si basalt or shale. 
R.W. MURRAY ET AL.

As with many aspects of analytical geochemistry, a balance exists between these parameters. The dilution factor from the procedure described herein should yield solutions that are stable at least for several days, and perhaps for up to several weeks.

\section{Interstitial Waters}

Diluted and undiluted interstitial waters are stable indefinitely, particularly if they are acidified. The most common contributing factor to a solution's demise is evaporation. All solutions must be stored tightly sealed (e.g., with a Parafilm gasket between the cap and the solution) and refrigerated, if possible. Samples should not be analyzed, however, until they have returned to room temperature.

\section{Drift Solution}

It is necessary to analyze a drift solution multiple times throughout an analytical run (see "Setting Up A Typical Analytical Run," p. 14). This solution will be used to identify and correct for instrumental drift. Typical drift observed for pore waters and igneous rock analyses during Leg 189 and the Leg 189 transit is on the order of 1\%-2\% per hour.

There are two important considerations in selecting a drift solution. (1) The drift solution must be matrix matched to the samples and standards that are being analyzed. If the matrix of the drift solution does not match the matrix of the samples and standards, then the behavior of the ICP-AES throughout the day may differ for the two different matrices, and thus the quantified drift would not accurately reflect variations in the sample analysis. (2) There must be an adequate concentration of target elements in the drift solution so the instrument can accurately quantify the intensity of each element.

It is not necessary to know the exact concentration of each element in the drift solution; however, it is critical that the composition of this solution remain uniform throughout the run. The analyst will not use the element concentrations during data reduction but rather the relative change in response to each element. This indicates the change in the ability of the ICP-AES to consistently measure these concentrations throughout the run.

\section{Rocks and Sediments}

The best drift monitors are samples that have been previously analyzed and are no longer needed. Thus, such finished solutions should be combined into a larger storage container for use as drift solution in the future. Solutions that have become unstable and thus unsuitable for quantitative analysis can also be added to this drift solution, provided they have been filtered to remove any gel that has formed and that no further gels are forming. Drift solutions are also useful, for example, to supply experimental solutions for nebulizer testing, checking or adjusting instrument parameters, setting up an analytical run, and other diagnostic purposes. Because of the importance of matrix matching, it may be advisable to keep separate drift solutions for carbonate-rich sediments, mid-ocean-ridge basalts (MORBs), shaley-type sediments, arc lavas, etc. There is no need to oversegregate, however, because the dominant matrix component is $\mathrm{LiBO}_{2}$ in all cases. Finally, even in natural samples, if the concentration of any element is too low, the analyst 
R.W. MURRAY ET AL.

should spike the drift solution with a single element standard of the low element so that the peak is easily detected by the ICP-AES.

\section{Interstitial Waters}

Because of the limited availability of interstitial water and its great value, unused sample solutions are likely to be archived or retained by the shipboard chemists. Thus, such solutions should not be used as drift solutions.

An artificial drift solution for interstitial waters can be constructed from filtered surface seawater. Recall that the concentrations need not be exact. To construct such a drift solution, the following equation can be used:

$$
\left(V_{1}\right)\left(C_{1}\right)=\left(V_{2}\right)\left(C_{2}\right)(1 / F W),
$$

where

$V_{1}=$ volume of drift solution to be prepared, in liters.

$C_{1}=$ target concentration of element in drift solution, in micromoles per liter.

$V_{2}=$ volume of single-element standard (VWR, Fisher, etc.) to add to solution, in milliliters.

$C_{2}=$ concentration of single-element standard supplied by vendor, in micrograms per milliliter.

$\mathrm{FW}=$ formula weight of element, in micromoles per microgram.

For example, most single element standard solutions are supplied at a concentration of $1000 \mu \mathrm{g} / \mathrm{mL}\left(C_{2}\right)$. To prepare $1 \mathrm{~L}$ of drift solution $\left(V_{1}\right)$ containing $\mathrm{Fe}$ at a concentration of $10 \mu \mathrm{mol}\left(C_{1}\right)$ (to mimic a $10 \times$ dilution of pore water that would have an undiluted concentration of $100 \mu \mathrm{mol})$, equation 1 is set up as follows:

$$
(1 \mathrm{~L})(10 \mu \mathrm{mol} / \mathrm{L})=\left(V_{2} \mathrm{~mL}\right)(1000 \mu \mathrm{g} / \mathrm{mL})(1 / 55.8 \mu \mathrm{mol} / \mu \mathrm{g}) .
$$

Solving the equation for $V_{2}$ results in $0.6 \mathrm{~mL}$ of Fe single-element solution to be added to prepare the 1-L drift solution. In practice, the best way to do this is to take $100 \mathrm{~mL}$ of filtered surface seawater, add $900 \mathrm{~mL}$ of DI (to yield $1 \mathrm{~L}$ ), and then spike this solution with $0.6 \mathrm{~mL}$ of 1000 $\mu \mathrm{g} / \mathrm{mL}$ Fe standard solution. Although this will result in a total volume of $1000.6 \mathrm{~mL}$, the deviation from exact volume is not significant enough to affect the utility of this solution as a drift monitor (which need not be quantitative).

Equation 1 can be applied for each element commonly analyzed by ICP-AES ( $\mathrm{Li}, \mathrm{B}, \mathrm{Fe}, \mathrm{Mn}, \mathrm{Sr}$, and $\mathrm{Ba}$ ), and a single multielement drift solution can be prepared. B, Li, and Sr do not need to be added to the artificial drift solution because their concentrations in seawater are high enough to be measured without an additional spike. Spiking with only the small amount of $\mathrm{Ba}$ indicated in Table $\mathrm{T} 2$ does not cause precipitation of barite $\left(\mathrm{BaSO}_{4}\right)$.

Table T2 provides the calculated results (from Equation 1) for preparation of a comprehensive artificial interstitial water drift solution. A companion Excel spreadsheet to Table T2, complete with linked Excel formulas and explanatory annotations, has been provided to ODP to assist in the construction of this drift solution.
T2. Construction of interstitial water drift solution, p. 25. 
R.W. MURRAY ET AL.

\section{Rinse Solution and Rinse Times}

It is necessary to rinse the sample uptake tubing and torch glassware between each sample and each standard to prevent carryover from the previous sample or standard. Rinsing also helps combat nebulizer clogging. The critical parameter of constraint is that the acid concentration of the rinse should be the same as the acid concentration of the samples and standards. This ensures that the plasma is not exposed to different matrices spaced closely together, which would negatively affect instrument stability throughout the run.

\section{Rocks and Sediments}

For the $\mathrm{LiBO}_{2}$ matrix, a $10 \% \mathrm{HNO}_{3}$ rinse solution works very well. A typical rinse time is $\sim 1.5 \mathrm{~min}$ between each sample or standard. This is a minimum time; it is in the analyst's best interest to use an adequate rinse time because the analytical run will be of higher quality and there will be less buildup of deposits on the torch glassware. Longer rinse times result in improvement of (i.e., reduction of) instrumental drift throughout the run.

\section{Interstitial Waters}

There is essentially no acid in the interstitial water samples, because the minimal acid amounts added during acidification of the initial squeezed sample is not enough to be of concern. However, rinsing with DI alone does not adequately keep the nebulizer orifice free of precipitating salts. A weak acid rinse $\left(\sim 0.5 \% \mathrm{HNO}_{3}\right)$ performs very well and does not present a sufficient matrix difference to adversely affect the analysis. Again, rinse times should be $\sim 1.5 \mathrm{~min}$ between each sample and standard.

\section{Analytical Wavelengths and Element Menus}

Wavelength selection is somewhat of an individual choice that commonly varies from analyst to analyst. However, there is a developing consensus regarding the wavelengths best suited for a particular target analyte. The wavelengths provided in Tables T3 and T4 have yielded good results in a variety of studies but should be considered to be suggestions only. If a particular shipboard scientist wishes to select a different wavelength that he or she has had better success with in the analysis of some type of sample, this can easily be accommodated by the JY2000 software. In the ICP-AES literature on board the ship, most of the publications suggest wavelengths appropriate for geological matrices, not only for the elements included here but also for others that a scientist may be interested in analyzing.

\section{Rocks and Sediments}

Appropriate wavelengths for ICP-AES analysis of rocks and sediments are listed in Table T3.
T3. Suggested wavelengths for ICP-AES analysis of rocks and sediments, p. 26.

T4. Suggested wavelengths for ICP-AES analysis of interstitial waters, p. 27. 
R.W. MURRAY ET AL.

Interstitial Waters

For interstitial waters, use of spectrophotometric procedures (Gieskes et al., 1991) is preferred for the analysis of $\mathrm{P}$ and $\mathrm{Si}$, particularly for those samples with relatively low concentrations. Similarly, ion chromatography (IC) is preferred for the analysis of $\mathrm{Ca}, \mathrm{Mg}$, and $\mathrm{K}$ (with $\mathrm{Na}$ being calculated by charge balance). However, ICP-AES analyses of these elements can provide important confirmation of spectrophotometric or IC results and is also worthwhile if there are difficulties with these other instruments. Experiments conducted during Leg 189 for the major cations provided excellent results, and preliminary work during the Leg 189 transit documented that total S can potentially be analyzed by ICP-AES in interstitial waters as well.

For higher concentration elements (such as the major cations), greater dilution factors may be required so the intensity of the wavelength remains within photomultiplier range. Additionally, to conserve sample, the greatest dilution possible should be used.

In summary, routine ICP-AES analyses can be relatively easily performed on interstitial water samples for $\mathrm{Fe}, \mathrm{Mn}, \mathrm{Sr}, \mathrm{Ba}, \mathrm{B}$, and $\mathrm{Li}$, with other elements added or deleted as deemed appropriate by the shipboard scientists and technical staff. These six elements can be determined on a single $10 \times$ dilution, based on $0.5 \mathrm{~mL}$ of sample and $4.5 \mathrm{~mL}$ of DI. Suggested wavelengths appropriate to the analysis of interstitial waters are given in Table T4.

\section{Calibration Standards}

ICP-AES is a comparative analytical technique, in that the instrument response (measured in "counts" units) must be calibrated against standards in which the concentrations of the various elements are known. There are two main calibration methods: (1) calibration against internationally recognized and approved standard reference materials (SRMs), or (2) calibration against synthetically prepared calibration standard solutions that have been constructed to closely mimic the matrix of the unknown samples. Each method has its strengths and weaknesses.

\section{Rocks and Sediments}

Calibration is best achieved through comparison to SRMs in a similar manner to that used in XRF analysis; however, whereas the XRF can retain a given calibration for several months, the ICP-AES must be calibrated before each analytical run. Selection of appropriate SRMs for calibration is dependent on the anticipated range of concentrations in the samples. Unlike the XRF, which is commonly calibrated on the basis of tens of individual SRMs, for ICP-AES analysis, robust linear calibration can be achieved with five SRMs and a blank. Because $\mathrm{LiBO}_{2}$ is the dominant matrix component, SRMs of differing lithologies can be used in the same calibration. For example, a single calibration composed of shale, andesite, basalt, and obsidian SRMs yields strong linearity for many elements. It is essential that the SRMs used for calibration be prepared with exactly the same TDS and acid concentration as the samples.

Because standard calibrations are required for each run, SRMs tend to be consumed relatively rapidly. In the interest of conservation, if there are enough calibration solutions left over from a previous run, and if 
R.W. MURRAY ET AL.

these solutions were prepared identically, then it is appropriate to reuse those SRM solutions until they are depleted.

It is common for laboratories to develop their own in-house calibration powders, to ease consumption of the often expensive and analytically valuable SRMs. The ODP laboratory should build a library of calibration standards using available rocks and sediments. After several preparations and analyses of these in-house samples, they can then be used for calibration purposes, as long as at least one independent certified SRM is analyzed as an unknown in each run to verify the calibration.

\section{Basalts}

We recommend calibration using the SRMs abbreviated as DNC-1, BIR-1, BHVO-2, W-2, and BCR-2. Additional well-characterized rocks (although not certified SRMs) used to check calibration results include K1919 and BAS140. For other igneous rock types, the above suite of SRMs can be modified to bracket the anticipated dynamic range of the data set.

\section{Sediments}

Variability in sedimentary compositions precludes use of a single set of SRMs to cover all sediment ranges. The three end-member compositions of sediments are shales, carbonates, and siliceous deposits. At this time, ODP has only a few sedimentary SRMs available. For shales, MAG1 , SCo-1, and other shales such as BCSS-1 or MESS-1, used in combination with high silica basalts will usually suffice. For carbonates, inclusion of NIST-1C (an argillaceous limestone) and other carbonate SRMs is essential. Variations in $\mathrm{Ca}$ are widely known to cause matrix effects, so care must be taken to select appropriate SRMs. Depending on the lithology of the targeted unknowns, a calibration using a blank (as a zero point), and two or three carbonate SRMs works moderately well. For siliceous sediments, use of rhyolite, as a high-silica standard, along with shale and basalt SRMs, works adequately.

\section{Interstitial Waters}

Calibration standards for interstitial waters must be constructed by the analyst, because an extended suite of seawater SRMs does not exist. Such standards can be prepared using one of the two following similar methods. In the first (less preferred) method, a spiked International Association for the Physical Sciences of the Ocean (IAPSO) solution is used as a master standard from which serial dilutions (approximately matrix matched to seawater by diluting with the synthetic seawater of Gieskes et al. [1991]) are prepared for eventual analysis. This technique has the advantage that the concentrations of the elements in the standards are exactly known, although the matrix match is not ideal. In the rare circumstances where the surface seawater method described below is not appropriate (low salinity due to rain or river input), this IAPSO method can be used to construct standards, although it is somewhat expensive.

The second approach (recommended here) is to use filtered surface seawater as the primary matrix, spiked accordingly to create a master standard solution from which serial dilutions in seawater are prepared. Surface seawater can be assumed to have concentrations of $\mathrm{Li}=25 \mu \mathrm{M}$, $\mathrm{B}=416 \mu \mathrm{M}$, and $\mathrm{Sr}=90 \mu \mathrm{M}$ (Millero, 1996), and the analyst can easily verify by ICP-AES that the concentrations of $\mathrm{Fe}, \mathrm{Mn}$, and $\mathrm{Ba}$ (found in nanomolar concentrations in surface seawater [Millero, 1996]) are es- 
R.W. MURRAY ET AL.

sentially zero. If dissolved silica will be measured by ICP-AES, the concentration in the surface water standard can be determined spectrometrically. This technique using surface seawater to construct the intermediate standard has the advantage that the matrix match is very robust but has the disadvantage that the initial concentrations of $\mathrm{Li}, \mathrm{B}$, and $\mathrm{Sr}$ (given above) may not be precise. However, in practice, this limitation is deemed to be minimal and can be easily verified using standard additions. The IAPSO solution can also be used as an appropriate check of accuracy.

For the preparation of these standards, we have provided ODP with a detailed series of numbered steps and explanations in spreadsheet form. Using the linked Excel formulas throughout the spreadsheet, the analyst can vary a number of parameters to construct different volumes and concentrations as needed.

\section{SETTING UP A TYPICAL ANALYTICAL RUN}

Described here is the process that is usually performed on a daily basis when the ICP-AES is used to perform a series of quantitative analyses. Because of the instrument's ability to provide rapid analysis, it is most efficient to analyze 20-25 samples per run. Along with calibration SRMs, drift solutions, blanks, replicate samples, calibration verifications, and other items, these tasks will fill an analytical day.

Additionally, for reasons relating to good analytical practice, this recommended general number of samples also makes sense-analytical precision is optimal when as many samples from a given site/hole are analyzed at the same time. For interstitial waters, samples from an entire site can be analyzed for a number of different trace elements in one run.

In all situations, it is advisable to analyze samples in random order (i.e., not in order of depth). Although analytical drift is accommodated throughout the run (see "Data Reduction," p. 17) analyzing samples in random depth order ensures that the final observed downhole trends are not caused artificially. All samples should analyzed at room temperature. Aspirating cold solutions (e.g., interstitial waters from the refrigerator) leads to high relative standard deviations and increased instrument drift.

The following descriptions apply to rocks and sediments as well as to interstitial waters and assumes that the method has been developed (complete with background corrections, selection of analytical mode, and all other parameters), calibration standards are constructed, samples have been diluted and are ready to run, and the sample file has been built. Some of the terminology may be unique to the JY instrument.

1. Warm up for at least 30 minutes. Do not be tempted to rush this. The warm-up period is not only for the generator but also for the glassware, torch box, and other aspects of the sample introduction system. Be sure to aspirate DI during the warm-up period. It is commonly helpful to aspirate a solution of the same matrix as the samples for the last $\sim 5$ min of the warm-up period. The plasma should not be run dry for long periods of time (exceeding $3 \mathrm{~min}$ ). A representative start-up procedure is provided in "Appendix," p. 22. 
R.W. MURRAY ET AL.

2. Perform a zero-order check, if needed. Zero order is the term used to define when the grating within the spectrometer behaves as a mirror, reflecting incoming light rather than refracting it into several wavelengths. A zero-order check physically moves the diffraction grating to its zero position, where all light is reflected. Although the software will check whether zero order is okay or not whenever the instrument is turned on, this internal software check is not a true mechanical test. It is instead a test of whether the electronics have been compromised (i.e., turned on or off, been subjected to a surge, etc.) since the ICP was last used. Therefore, it is advisable to perform a zero-order check (Shift-F2; Center Control) every few days. It is also helpful to record in a logbook at what position (step number) zero order was found, as this parameter is useful for diagnostics, should the need arise.

3. Perform an autosearch. During elemental analysis (e.g., Fe at $238.204 \mathrm{~nm}$ ), the spectrometer searches for a reference peak (the carbon line at $193.031 \mathrm{~nm}$ ) and subsequently moves to the chosen wavelength as a function of its distance (in motor steps, which corresponds to $\mathrm{nm}$ ) from the reference peak. Prior to each analysis, the ICP searches for the reference peak and locates it. The autosearch function calibrates the instrument exactly for each peak location with respect to the reference, so during analysis it can locate the wavelength precisely. The autosearch also quantifies an offset, which is the distance (in nanometers) that the reference peak was found from where it was expected. Typical offsets are on the order of $0.005 \mathrm{~nm}$ or less. Perform autosearch repeatedly until the peaks are visually on-line each time, paying attention to the amount of offset (in nanometers). Offsets significantly and consistently greater than $\sim 0.005 \mathrm{~nm}$ may indicate something is amiss (in practice this will depend on ODP's exact instrument). We recommend performing several autosearch commands in a series immediately prior to calibration. The first time an element is autosearched, or whenever a method has been changed significantly, be sure to use a single element solution to ensure that the peak found during an autosearch is in fact a peak for the element of interest. Subsequently, a standard or drift solution can be used to Autosearch. Using the Small Window option on the JY2000 software also helps ensure that the peak found is the one of interest.

4. Perform a calibration. If the calibration feature of the software is chosen, the instrument must be calibrated for every analyte of interest prior to each run (see "Constructing a Sample File and Performing Data Reduction," p. 16, for details on calibration options). The operating conditions of the ICP during the calibration must be identical to analysis conditions, so all manipulation of gas flows, pump speeds, and the like must have taken place prior to this stage. In fact, if any operating conditions have changed, a new Autosearch will need to be performed. Using the autosampler helps ensure that the calibration conditions (e.g., rinse time) will be identical. Note: the JY2000 software does not allow for correction of drift between calibration standards, so the analyst is forced to assume that only minimal drift takes place during this aspect of a run. This assumption has been shown to be relatively robust and its effects minimized during data reduction.

5. Start Run. Initiation of the analytical run must occur as soon as possible after the calibration is completed (usually within 5-10 min). This is of vital importance because the first item in the sample file is a drift solution, which will be used as the master drift to correct the entire run. Provided the sample file is ready, the autosampler is loaded, and all 
R.W. MURRAY ET AL.

other preparations are completed, starting the run this quickly is not difficult.

\section{CONSTRUCTING A SAMPLE FILE AND PERFORMING DATA REDUCTION}

Because the mechanics of data reduction are a direct result of the order that standards, unknowns, drift solutions, and other standard reference materials are analyzed, it is most appropriate to consider sample file and data reduction together.

There is no single correct method to reduce the data acquired during an ICP run. The discussion below outlines several alternatives and is not a comprehensive list. Each of these procedures has been demonstrated to work well. This section is intended to outline some of the general principles and goals of data reduction. We have provided to ODP specially designed Excel spreadsheets to perform these calculations in a fashion that is based on results from Legs 187-189 and at Boston University. These spreadsheets are located onboard the Resolution.

The one commonality to all data reduction methods is that the analyses of one or more SRMs in the analytical run, when treated as unknown samples, yields a result that is in agreement with the internationally recognized values. SRMs can be used in the analysis of rocks and sediments, and IAPSO seawater can be used (for some elements) in the analysis of interstitial waters. For Fe, Mn, and $\mathrm{Ba}$ (those elements not contained in IAPSO solution), accuracy can be assessed by comparison to a spiked IAPSO sample.

\section{Sample File}

A sample file is constructed by the analyst and is accessed by the ICP and the autosampler to analyze a series of analytes, including unknown samples, drift solutions, blanks, and any other solutions desired. In addition to specifying the physical position of each solution in the autosampler rack, the sample file tells the ICP the order in which these solutions are analyzed. A sample file is not used for calibration. For details on how to build a sample file using the JY software, please refer to the companion "Software Notes" that are located on the Resolution.

The critical component in the sample file is the order in which the samples are run, for this will affect the ability to perform an appropriate data reduction. A typical sample file may look like the following (note that this would be for a short run; a total of up to 30-40 items is more typical):

Drift 1

SRM-1

Drift 2

Sample A

Sample B

Drift 3

SRM-2

Sample C

Sample D

Drift 4

SRM-3 
R.W. MURRAY ET AL.

ZIP

Sample E

Drift 5, and so on...

There are several critical aspects to this sample file:

1. The run starts and finishes with a drift solution.

2. Drift solutions are run more frequently at the beginning of the run, when the ICP is more prone to instability.

3. Drift solutions should be run no less frequently than every 20$30 \mathrm{~min}$ of instrument time after the first few drifts have been run. Thus, the frequency of drift analyses depends on how many elements are being analyzed in that particular method.

4. The item identified as ZIP is a procedural blank. Avoid use of the word Blank or Blk in the Sample File, as well as of Standard or Std, as these at times have specific meaning to the JY software and may cause an unintended data manipulation.

5. Standards and check solutions are spaced throughout the run. For the analysis of rocks and sediments, these may be SRMs; for the analysis of interstitial waters, these may be calibration standards rerun as part of the Sample File, as well as Ba calibration standards.

\section{Data Reduction}

There are two main approaches to calibration and data reduction. We have tested calibration routines for both interstitial waters and igneous rocks using the calibration software (Option 1, below). Accordingly, the spreadsheets we have provided to ODP are tailored to Option 1.

1. Using the calibration feature. If a calibration is performed immediately prior to launching the Sample File, the JY software will provide concentration data for each item as the run proceeds. Thus, the first steps of the data reduction are performed by the JY software. Although the concentrations calculated during the run are preliminary because they have not been drift corrected, the analyst can immediately determine if the data appear to be reasonable as the run proceeds. If not, the run can be terminated and an explanation sought. The final data can be drift corrected using the Excel spreadsheets provided to ODP.

2. Without use of calibration feature. If the JY calibration software option is not selected, the analyst can construct a sample file similar to one shown above and perform all data reduction offline using the following steps.

a. Drift correct all counts data, by assuming a constant linear change between drifts.

b. Blank subtract, by subtracting the counts of each element in the Zip item from each unknown.

c. Construct a calibration line for each element, based on the drift-corrected, blank-subtracted counts plotted on an $x-y$ graph against the known concentration of the SRM (or in the case of interstitial waters of the synthetically constructed standard). Because the items were blank subtracted, the calibration line can be forced through the origin $(0,0)$ if desired. 
R.W. MURRAY ET AL.

d. Calculate the concentration of each element in each unknown, using the equation of the calibration line derived above.

The main advantages of Option 2 are that it gives the analyst flexibility in all aspects of data reduction and it is not affected by the assumption of zero drift during the calibration because the SRMs used in the calibration are drift corrected along with the samples. In addition to being very time consuming, the main disadvantage to the manual data reduction of Option 2 is that the analyst can not assess if a run has been successful until the run is completed and all the data have been reduced. As solutions are somewhat precious and there is commonly a delay between a series of data acquisition runs and the associated data reduction, this often is not desirable.

\section{BASIC MAINTENANCE SUGGESTIONS}

\section{Unclogging a Nebulizer}

Sometimes in the course of human events a nebulizer will become clogged. Clogging can be diagnosed by a steady decrease in the Ar flow rate with a corresponding rise in the Ar backpressure. Additionally, the stability of the instrument will greatly decrease. Because of the importance of the nebulizer to the analytical process, great care must be taken to be prevent clogging.

The best way to unclog a nebulizer is to never let it clog in the first place. This can be accomplished by filtering all samples, standards, drifts, and any other solution that is aspirated. Keep the wash bottle, rinse bottle, and other solutions covered whenever possible. If a widemouth bottle is used for the rinse bottle, boring a small hole in the cap will minimize the input of dust into the solution.

Never use an ultrasonic bath to unclog a nebulizer. The vibrations in such a bath will harm the delicate glass channel of the nebulizer. Because the channel of a concentric nebulizer gets successively more narrow towards the tip, never try to clean a nebulizer by forcing gas or water in the forward direction.

Soaking the nebulizer in $20 \%$ (or greater) $\mathrm{HNO}_{3}$ or in aqua regia solution (1 part $\mathrm{HNO}_{3}: 3$ parts $\mathrm{HCl}$ ) greatly assists the unclogging process. Inject the acid solution (wearing gloves) into the front of the nebulizer, and also gently inject some acid up from the Ar channel. This will ensure that the clogged tip will be inundated from all directions. Such injection can be achieved by outfitting a syringe with the appropriate sized Tygon tubing. Let the nebulizer soak overnight, in a heated bath if possible. Additionally, provided the nebulizer has been thoroughly rinsed with deionized water (and thus there is no remaining acid present), the nebulizer can be sometimes unclogged by unhooking one of the Ar gas flow lines from the ICP torch assembly, placing the end of the tube over the tip of the nebulizer, and using the high Ar pressure to blow back through the nebulizer. Be sure to hold the nebulizer tightly to prevent it from launching across the room. 
R.W. MURRAY ET AL.

ANALYSIS OF MAJOR AND TRACE ELEMENTS BY ICP-AES

\section{Cleaning the Quartz Glassware and Alumina Injector Tip}

The fully demountable torch assembly from JY lends itself to easy cleaning. Precipitate will build up on the surfaces of the outer tube, inner tube, and alumina injector. This is detrimental to the analysis because it affects plasma shape and stability and inhibits analyte ionization. Depending on the concentration of acid and the dilution factor of the $\mathrm{LiBO}_{2}$ fusions, the quartz tube surfaces will chip and abrade. For the fused rock and sediment samples, it is recommended to clean the glassware frequently (after $2-4$ analytical runs) to help maintain instrument stability and minimize glassware corrosion.

There is a difference between precipitate buildup and staining. Staining is inevitable and can be tolerated. Precipitate on the surfaces of the glassware, however, is more serious and must be monitored. The key issue is that the glassware must be smooth, with the unchipped and high-quality edges. The outer quartz tube must be very clean with minimal stains.

Soaking all glassware in $20 \%$ (or stronger) $\mathrm{HNO}_{3}$ or aqua regia solution helps the cleaning process. As with the nebulizer, let the glassware soak overnight, in a heated bath if possible. Avoid the use of an ultrasonic bath. Use KimWipes or other clean lint-free cloths to polish the outside of the tube, and pass the KimWipe through the inside of the cylinder and pass back and forth.

The inner quartz tube can be inverted (reversed) so the stained end is at the bottom of the torch assembly, but if this is done be sure that the edges of the tube are smooth and sharp so that the bottom of the tube is seated properly. Soaking the upper end of the alumina tip, even in relatively weak $\mathrm{HNO}_{3}$, helps clean away precipitate easily.

\section{USEFUL REFERENCES DISCUSSING ICP-AES TECHNIQUES}

The following articles provide excellent background information on many aspects of ICP-AES, including much information specific to the analysis of geological matrices. Copies of each of these articles are available in the Chemistry Laboratory on the drillship. Additionally, note that two of these articles come from a special issue of Chemical Geology that is devoted to ICP-AES and ICP-mass spectrometry (ICP-MS). Other articles in this issue (not listed here) may also be of interest.

Jarvis, I., and Jarvis, K.E., 1992a. Inductively coupled plasma-atomic emission spectrometry in exploration geochemistry. J. Geochem. Expl., 44:139-200.

- 1992b. Plasma spectrometry in the earth sciences: techniques, applications and future trends. Chem. Geol., 95:1-33.

Montaser, A., and Golightly, D.W., 1992. Inductively Coupled Plasmas in Analytical Atomic Spectrometry: New York (VCH Publ.).

Potts, P.J., 1987. Inductively coupled plasma-emission spectrometry. In Potts., P.J. (Ed.), A Handbook of Silicate Rock Analysis: London (Blackie Academic and Professional), 153-197.

Totland, M., Jarvis, I., and Jarvis, K.E., 1992. An assessment of dissolution techniques for the analysis of geological samples by plasma spectrometry. Chem. Geol., 95:35-62. 
R.W. MURRAY ET AL.

Walsh, J.N., and Howie, R.A., 1986. Recent developments in analytical methods: uses of inductively coupled plasma source spectrometry in applied geology and geochemistry. Appl. Geochem., 1:161-171.

\section{ACKNOWLEDGMENTS}

In the construction of this Technical Note we have drawn heavily on the expertise and input of a number of different individuals and institutions. At Boston University, we particularly thank Terry Plank, Katie Kelley, Lauren Vigliotti, Joel Sparks, and Eric Grunwald for their feedback and assistance during the refinement of some of the methodologies described herein. We also thank Gilles Poncet and his colleagues at the Jobin-Yvon Emission Division of Instruments SA, Inc., for their responsiveness and assistance. At the Ocean Drilling Program, the efforts of Dennis Graham, Chieh Peng, Anne Pimmel, Jaque Ledbetter, Brad Julson, Tim Bronk, Don Sims, Johanna Suhonen, and other members of the scientific and technical staff eased the transition of installing and erating the ICP-AES device on board the ship during Legs 187-189.

The scientific community responded positively to this installation and development effort, and in particular we acknowledge the support of the Leg 185 igneous and pore-water scientists, as well as Eric DeCarlo, Dave Christie, Vaughn Balzer, Mitch Malone, and other members of the Legs 187-189 scientific and technical parties.

We acknowledge the support of the U.S. National Science Foundation and the U.S. Geological Survey of the Boston University Analytical Geochemistry Facility, where protocols were tested for the ODP shipboard environment. We also thank the USDepartment of Energy for financial support associated with the acquisition of the ODP shipboard ICP-AES instrument. 
R.W. MURRAY ET AL.

\section{REFERENCES}

Gieskes, J.M., Gamo, T., and Brumsack, H., 1991. Chemical methods for interstitial water analysis aboard JOIDES Resolution. ODP Tech. Note, 15.

Millero, F. J., 1996, Chemical Oceanography (2nd ed): New York (CRC Press).

Shipboard Scientific Party, in press a. Explanatory notes. In Christie, D.M., Pedersen, R.B., Miller, D.J., et al., Proc. ODP, Init. Repts., 187 [CD-ROM]. Available from: Ocean Drilling Program, Texas A\&M University, College Station TX 77845-9547, USA.

Shipboard Scientific Party, in press b. Explanatory notes. In Cooper, A.K., O'Brien, P.E., Richter, C., et al., Proc. ODP, Init. Repts., 188 [CD-ROM]. Available from: Ocean Drilling Program, Texas A\&M University, College Station TX 77845-9547, USA.

Shipboard Scientific Party, in press c. Explanatory notes. In Exon, N.F., Kennett, J.P., Malone, M.J., et al., Proc. ODP, Init. Repts., 189 [CD-ROM]. Available from: Ocean Drilling Program, Texas A\&M University, College Station TX 77845-9547, USA. 
R.W. MURRAY ET AL.

\section{APPENDIX}

\section{Start-Up Procedure}

1. Empty drain bottle if needed.

2. Check peristaltic pump tubing is not flattened. Replace if necessary.

3. Turn on cooling water.

4. Turn on exhaust fan.

5. Turn on argon at tank/regulator.

6. Turn on peristaltic pump and begin pumping DI water.

Operating Conditions

Plasma flow: $12-14 \mathrm{~L} / \mathrm{min}$

Pump speed: 20

Nebulizer pressure: $\sim 3$ bar

Argon humidifier: ON for basalts and fusions, OFF for interstitial waters

7. Pump DI at least 5 min prior to ignition. Check IN and OUT flow.

8. Initiate start-up procedure through the Windows software.

9. Recheck plasma.

10. Let instrument warm up for at least one-half hour...

\section{Shut-Down Procedure}

1. Pump rinse solution for at least $5 \mathrm{~min}$.

2. Pump DI for at least 3 min.

3. Initiate stop procedure through the Windows software.

4. Maintain gas flow and pump DI for 3 min.

5. Remove input tubing from DI and pump until nebulizer and spray chamber are dry.

6. Turn peristaltic pump off, release clamp, and unhook pump tubing.

7. Turn off recirculating water. 
R.W. MURRAY ET AL.

ANALYSIS OF MAJOR AND Trace ELEMENTS BY ICP-AES

\section{PUBLISHER'S NOTES}

Material in this publication may be copied without restraint for library, abstract service, educational, or personal research purposes; however, this source should be appropriately acknowledged.

Murray, R.W., Miller, D.J., Kryc, K.A., 2000. Analysis of major and trace elements in rocks, sediments, and interstitial waters by inductively coupled plasma-atomic emission spectrometry (ICP-AES). ODP Tech. Note, 29 [Online]. Available from World Wide Web: <http://www-odp.tamu.edu/publications/tnotes/tn29/ INDEX.HTM>. [Cited YYYY-MM-DD]

Distribution: Electronic copies of this publication may be obtained from the ODP Publications homepage on the World Wide Web at: http://www-odp.tamu.edu/publications.

This publication was prepared by the Ocean Drilling Program, Texas A\&M University, as an account of work performed under the international Ocean Drilling Program, which is managed by Joint Oceanographic Institutions, Inc., under contract with the National Science Foundation. Funding for the program is provided by the following agencies:

Australia/Canada/Chinese Taipei/Korea Consortium for Ocean Drilling

Deutsche Forschungsgemeinschaft (Federal Republic of Germany)

Institut National des Sciences de l'Univers-Centre National de la Recherche Scientifique (INSU CNRS; France)

Ocean Research Institute of the University of Tokyo (Japan)

National Science Foundation (United States)

Natural Environment Research Council (United Kingdom)

European Science Foundation Consortium for Ocean Drilling (Belgium, Denmark, Finland, Iceland, Ireland, Italy, The Netherlands, Norway, Portugal, Spain, Sweden, and Switzerland)

Marine High-Technology Bureau of the State Science and Technology Commission of the People's Republic of China

\section{DISCLAIMER}

Any opinions, findings, and conclusions or recommendations expressed in this publication are those of the author(s) and do not necessarily reflect the views of the National Science Foundation, the participating agencies, Joint Oceanographic Institutions, Inc., Texas A\&M University, or Texas A\&M Research Foundation. 
R.W. MURRAY ET AL.

ANALYSIS OF MAJOR AND TRACE ELEMENTS BY ICP-AES

Table T1. Conversion factors between oxide and elemental concentrations.

\begin{tabular}{lcl}
\hline $\begin{array}{l}\text { Oxide } \\
(w t \%)\end{array}$ & $\begin{array}{c}\text { Conversion } \\
\text { factor }\end{array}$ & $\begin{array}{c}\text { Element } \\
(w t \%)\end{array}$ \\
\hline $\mathrm{SiO}_{2}$ & 0.4675 & $\mathrm{Si}$ \\
$\mathrm{Al}_{2} \mathrm{O}_{3}$ & 0.5293 & $\mathrm{Al}$ \\
$\mathrm{Fe}_{2} \mathrm{O}_{3}$ & 0.6994 & $\mathrm{Fe}$ \\
$\mathrm{FeO}$ & 0.7773 & $\mathrm{Fe}$ \\
$\mathrm{MnO}$ & 0.7745 & $\mathrm{Mn}$ \\
$\mathrm{TiO}_{2}$ & 0.5995 & $\mathrm{Ti}$ \\
$\mathrm{CaO}$ & 0.7143 & $\mathrm{Ca}$ \\
$\mathrm{Na}{ }_{2} \mathrm{O}$ & 0.7419 & $\mathrm{Na}$ \\
$\mathrm{K}_{2} \mathrm{O}$ & 0.8301 & $\mathrm{~K}$ \\
$\mathrm{MgO}$ & 0.6030 & $\mathrm{Mg}$ \\
$\mathrm{P}_{2} \mathrm{O}_{5}$ & 0.4365 & $\mathrm{P}$ \\
\hline
\end{tabular}


R.W. MURRAY ET AL.

ANALYSIS OF MAJOR AND Trace ELEMENTS BY ICP-AES

Table T2. Construction of interstitial water drift solution.

\begin{tabular}{|c|c|c|c|c|c|c|c|}
\hline Element & $\begin{array}{l}\text { Concentration in } \\
\text { undiluted sample } \\
\qquad(\mu \mathrm{M})\end{array}$ & $\begin{array}{l}\text { Concentration in } \\
\text { drift solution* } \\
(\mu \mathrm{M})\end{array}$ & $\begin{array}{l}\text { Formula weight** } \\
\qquad(\mu \mathrm{g} / \mu \mathrm{mol})\end{array}$ & $\begin{array}{l}\text { Volume of drift } \\
\text { solution***} \\
\text { (L) }\end{array}$ & $\begin{array}{l}\text { Concentration of } \\
\text { primary standard } \dagger \\
(\mu \mathrm{g} / \mathrm{ml})\end{array}$ & $\begin{array}{l}\text { Volume of primary } \\
\text { standard to add } \\
\text { (L) }\end{array}$ & $\begin{array}{l}\text { Volume of primary } \\
\text { standard to add } \dagger \dagger \\
(\mathrm{ml})\end{array}$ \\
\hline $\mathrm{Li}$ & 25 & 2.5 & 6.9 & 1 & 1000 & 0 & 0 \\
\hline B & 416 & 40 & 10.8 & 1 & 1000 & 0 & 0 \\
\hline $\mathrm{Mn}$ & 100 & 10 & 54.9 & 1 & 1000 & 0.0005 & 0.5 \\
\hline $\mathrm{Fe}$ & 100 & 10 & 55.8 & 1 & 1000 & 0.0006 & 0.6 \\
\hline $\mathrm{Sr}$ & 90 & 9 & 87.6 & 1 & 1000 & 0 & 0 \\
\hline
\end{tabular}

Notes: From "Equation 1," p. 10: ${ }^{*}=\mathrm{C} 1,{ }^{* *}=\mathrm{FW},{ }^{* *}=\mathrm{V} 1, \dagger=\mathrm{C} 2, \dagger \dagger=\mathrm{V} 2$. Concentration in drift solution $=10 \times$ dilution of concentration in undiluted sample. 
R.W. MURRAY ET AL.

ANALYSIS OF MAJOR AND TRACE ELEMENTS BY ICP-AES

Table T3. Suggested wavelengths for ICP-AES analysis of rocks and sediments.

\begin{tabular}{|c|c|c|}
\hline Element & $\begin{array}{l}\text { Wavelength } \\
\qquad(\mathrm{nm})\end{array}$ & Remarks \\
\hline Si & 251.611 & \\
\hline Al & 396.152 & \\
\hline $\mathrm{Ti}$ & 334.941 & This line is a doublet but is preferred \\
\hline $\mathrm{Ti}$ & 308.802 & \\
\hline $\mathrm{Fe}$ & 259.940 & \\
\hline Mn & 257.610 & \\
\hline $\mathrm{Ca}$ & 393.366 & \\
\hline $\mathrm{Mg}$ & 285.213 & \\
\hline $\mathrm{Na}$ & 589.592 & \\
\hline K & 766.490 & \\
\hline$P$ & 178.229 & This line requires $\mathrm{N}_{2}$ flush and is preferred \\
\hline $\mathrm{P}$ & 213.618 & \\
\hline $\mathrm{Zr}$ & 343.823 & \\
\hline Y & 371.030 & \\
\hline $\mathrm{Sr}$ & 407.771 & \\
\hline $\mathrm{Zn}$ & 213.856 & \\
\hline $\mathrm{Cu}$ & 324.754 & \\
\hline $\mathrm{Ni}$ & 231.604 & \\
\hline $\mathrm{Cr}$ & 267.716 & \\
\hline V & 292.402 & \\
\hline SC & 361.384 & \\
\hline $\mathrm{Ba}$ & 455.403 & \\
\hline
\end{tabular}


R.W. MURRAY ET AL.

ANALYSIS OF MAJOR AND TRACE ELEMENTS BY ICP-AES

Table T4. Suggested wavelengths for ICP-AES analysis of interstitial waters.

\begin{tabular}{lc}
\hline Element & $\begin{array}{c}\text { Wavelength } \\
(\mathrm{nm})\end{array}$ \\
\hline $\mathrm{Ba}$ & 249.773 \\
$\mathrm{Fe}$ & 259.940 \\
$\mathrm{Mn}$ & 257.610 \\
$\mathrm{Sr}$ & 407.771 \\
$\mathrm{Ba}$ & 455.403 \\
$\mathrm{Li}$ & 670.784 \\
\hline
\end{tabular}

